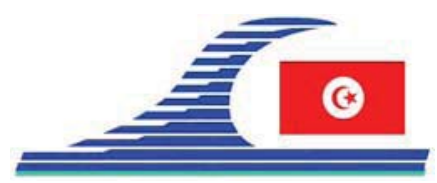

\author{
Conférence Méditerranéenne Côtière et Maritime \\ EDITION 1, HAMMAMET, TUNISIE (2009) \\ Coastal and Maritime Mediterranean Conference \\ Disponible en ligne - http://www.paralia.fr-Available online
}

\title{
Suivi de la dynamique littorale des plages de Corse : le ROL (Réseau d'Observation du Littoral corse)
}

\author{
Yann BALOUIN ${ }^{1}$, Eric PALVADEAU ${ }^{2}$, \\ Gwenaëlle BODERE $^{2}$, Vincent HENNEQUIN ${ }^{2}$
}

1. BRGM, Service Géologique du Languedoc-Roussillon, 1039 rue de Pinville, 34000 Montpellier, France.

y.balouin@brgm.fr

2. BRGM, Service Géologique de Corse, Z.I. de Furiani, 20600 Bastia, France.

\section{Résumé :}

En 1999, dans le cadre des accords entre l'Office de l'Environnement de la Corse et le BRGM, un Réseau d'Observation du Littoral de la Corse a été mis en place afin de fournir les données nécessaires à la compréhension et à l'identification des évolutions observées et des remèdes possibles. Son objectif est triple : (1) apprécier les évolutions du littoral et comparer les situations ; (2) fournir des éléments de prise de décision aux aménageurs régionaux et (3) fournir des bases techniques de prédiction (modélisation). Ce réseau s'étend tout à la fois à des sites témoins représentatifs des évolutions régionales naturelles, à des sites à évolutions critiques ponctuelles et à des sites économiquement sensibles ou soumis à l'impact d'aménagements.

Cette contribution fait le bilan des observations de 2000 à 2009, en termes d'évolution des sites suivis, et de dynamique littorale. Les résultats préliminaires des analyses en cours pour caractériser l'hydrodynamique et les processus de submersions marines seront également présentés.

\section{Mots-clés :}

Réseau d'observation - Dynamique sédimentaire - Submersion marine

\section{Introduction}

La Corse est sujette à un recul de son littoral du, soit à des phénomènes naturels (diminution des apports solides des fleuves, etc.), soit à des aménagements. Cette érosion peut avoir des conséquences économiques et/ou environnementales importantes. La régression des plages menace notamment les activités touristiques de stations balnéaires, mais également des zones de protection environnementales soumises à un aléa érosion / submersion marine croissant.

Depuis 1999 (PALVADEAU \& NAY, 2000), dans le cadre de la convention OECBRGM, un programme d'observation a été mis en place pour fournir les données nécessaires à la compréhension et à l'identification des évolutions observées et des remèdes possibles. Ce programme aboutit à un Réseau d'Observation du Littoral de la DOI: $10.5150 / \mathrm{cmcm} .2009 .021$ 
Corse qui s'étend à des sites témoins représentatifs des évolutions régionales naturelles, à des sites à évolutions critiques ponctuelles et à des sites économiquement sensibles à des impacts d'aménagements. L'objectif du Réseau d'Observation est de:

- Etre un outil de suivi du littoral : il a pour mission de valoriser et de compléter les informations existantes sur le littoral, de caractériser les évolutions des systèmes côtiers, d'évaluer les vitesses de recul du trait de côte, mais également la dynamique des morphologies sous-marines qui constituent le stock sédimentaire disponible;

- Etre un outil de mutualisation et d'organisation de l'information : Le réseau de suivi œuvre pour mutualiser les efforts de connaissance et d'acquisition de données d'évolution du littoral corse;

- Etre un outil de prospective : Le réseau d'observation a pour objectif de développer des outils de compréhension et de prospective visant à anticiper les grands changements sur le littoral.

La méthodologie mise en œuvre répond à un réel besoin de données pour une meilleure compréhension des processus d'évolution, mais elle constitue également un outil d'aide à la gestion du littoral corse permettant à la fois d'estimer la vulnérabilité des côtes, et de fournir les informations requises pour la définition des éventuelles stratégies d'aménagement et le dimensionnement des projets.

\section{Méthodologie}

Aujourd'hui, le Réseau d'Observation du Littoral comprend 15 sites. Il repose sur l'acquisition : (1) de profils de plage transversaux (33 profils de plage); et (2) de levés longitudinaux du trait de côte (total de $41 \mathrm{~km}$ ) (Figure 1). Ces différents levés sont réalisés à l'aide de GPS cinématique, assurant une précision centimétrique de la mesure. Les levés longitudinaux sont réalisés à l'aide d'un quad. Deux traits morphologiques sont ainsi repérés et géoréférencés : le pied de dune; la berme ou à défaut la ligne d'eau, niveau moyen de l'eau atteint en période de calme ou «trait de côte moyen ». Les levés bathymétriques sont réalisés à l'aide d'un GPS différentiel Trimble R6 à précision centimétrique couplé, en mer, à un sondeur bathymétrique par le logiciel Hypack ${ }^{\circledR}$. En 2006, un levé aéroporté LIDAR a été réalisé sur le littoral du lido de la Marana pour évaluer la pertinence de cet outil pour les observations. En 2009, un levé lidar est prévu sur l'ensemble du littoral de la plaine orientale.

\section{Bilan des observations 2000-2009}

Les suivis réalisés depuis 2000 fournissent de bons indicateurs de l'état des plages et permettent d'établir les tendances d'évolution des différents sites suivis (BALOUIN et al., 2009). Les plages ouvertes de la plaine orientale présentent des évolutions contrastées qui résultent principalement de l'action de la dérive littorale résultante vers le nord, et de la présence de morphologies sous-marines de barres souvent fortement tridimensionnelles. A l'échelle saisonnière, l'état de la plage est à mettre en relation avec 
des phénomènes transverses. En effet, la position des barres externes (qui reculent en hiver) est étroitement liée à la position du trait de côte. De même, les morphologies de barres en croissant affectent directement l'état de la plage avec des zones stables en face des cornes, et des zones où alternent érosion et accrétion en face des baies (voir exemple de Padulone, Figure 2).

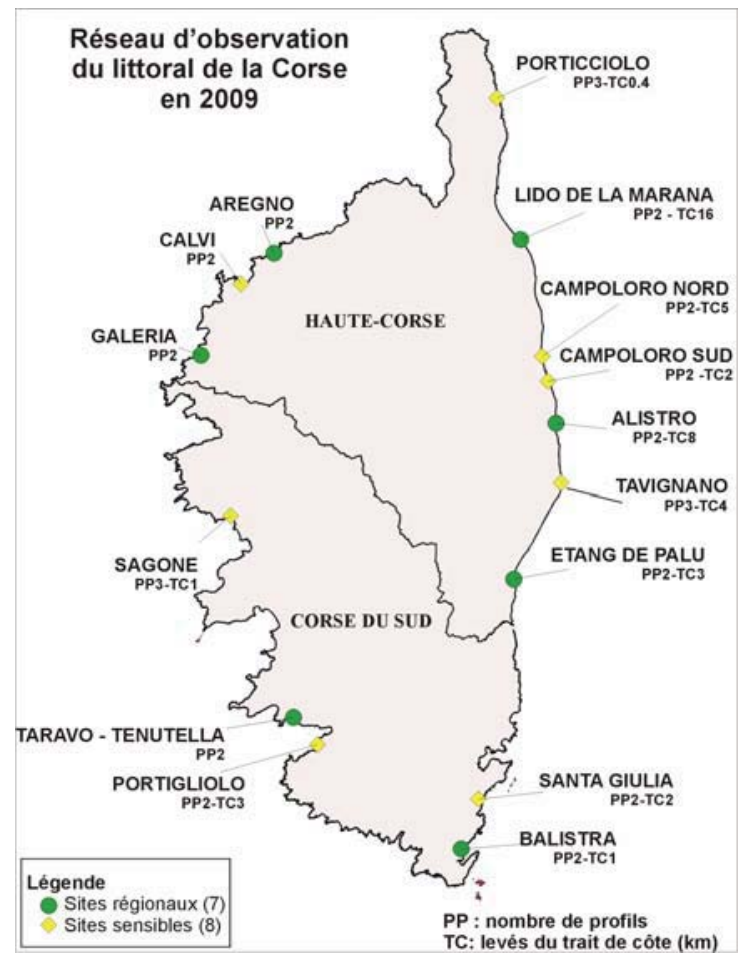

Figure 1. Le réseau d'observation du littoral de la Corse en 2009.

Les plages de baies ont un comportement différent selon leur exposition et leur géomorphologie. Ainsi, une plage à galets fortement réflective comme Galeria est soumise à des houles de forte intensité, mais présente une bonne stabilité depuis le début des levés. En revanche, les plages telles que Porticciolo ou Taravo-Tennutella, qui présentent un profil plus dissipatif et qui sont soumises à des houles de tempêtes importantes, montrent un recul faible mais continu de la plage, malgré des phases de récupération. La plage de Porticciolo est devenue extrêmement étroite et ne doit sa préservation qu'aux accumulations de feuilles de posidonies durant toute la période hivernale.

\section{Caractérisation des aléas érosion et submersion marine}

Afin de mieux comprendre le fonctionnement de ces sites et leur évolution, des études sont en cours pour caractériser l'hydrodynamique et les processus d'évolution. Cette analyse vise à caractériser l'aléa submersion marine et concerne principalement la plaine orientale de Corse qui reste le secteur le plus vulnérable. 
Les chroniques de houles ont pu être reconstituées à partir des champs de vents NCEP (1979-2009). Ces données, en cours de validation, permettront d'analyser les statistiques de la houle et de déterminer les épisodes les plus pénalisants pour le littoral. Pour cela, une base référençant tous les événements de tempêtes et tous les impacts recensés est en cours d'élaboration. Grâce au levé très haute précision LIDAR, des simulations de ces événements seront réalisées afin de caractériser l'aléa submersion marine. Les résultats préliminaires de ces analyses seront présentés.
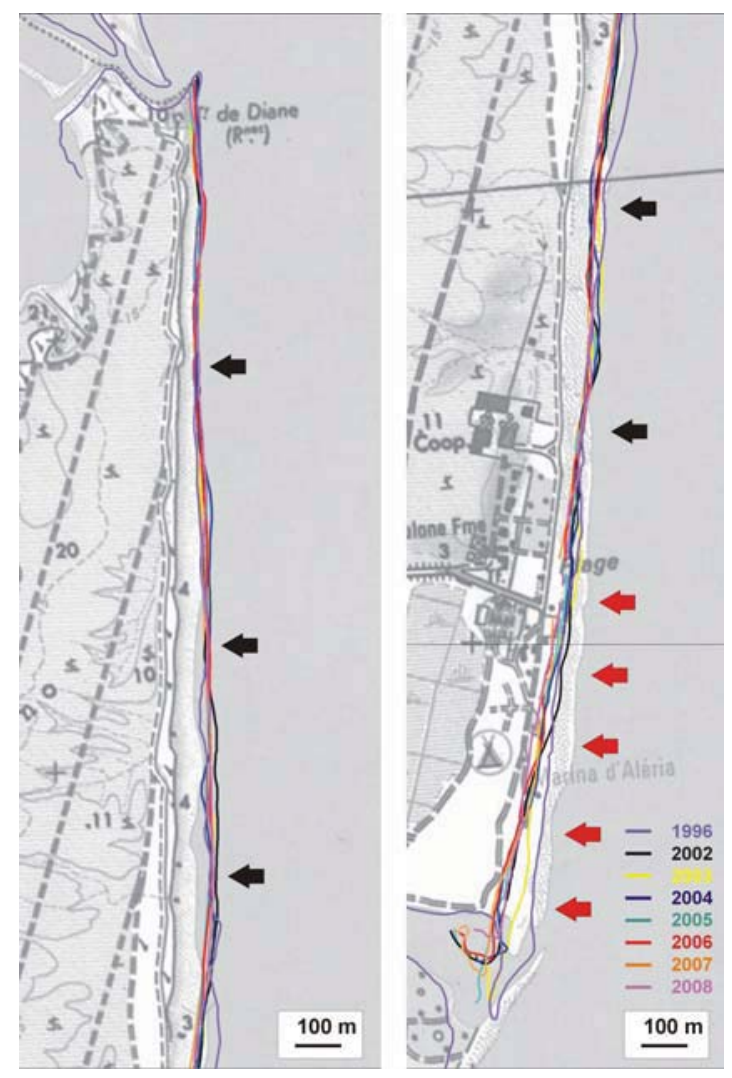

Figure 2. Positions du trait de côte sur le secteur de Padulone (Tavignano). Les flèches noires indiquent les secteurs "nœuds" où l'érosion reste modérée depuis 1996, les flèches rouges indiquent les secteurs où l'érosion est continue depuis 1996. La position de ces nœuds et ventres est étroitement liée à la position des morphologies sous-marines de barres en croissant. A gauche : secteur nord, à droite : secteur sud du site.

\section{Références bibliographiques}

BALOUIN Y., PALVADEAU E., BODERE G. (2009). Réseau d'observation $d u$ littoral de la Corse. Rapport d'observation 2008. BRGM/RR-57521-FR, 153 p.

PALVADEAU E., NAY K.M. (2000). Réseau d'observation du littoral de la Corse. Choix et implantation des sites. BRGM/RR-40965-FR, BRGM, Orléans. 